Technical note

\title{
Evaluation of medical physics training in radiology residency in 67 countries
}

\author{
Madan M. Rehani ${ }^{\mathrm{a}, *}$, Ruben Pauwels ${ }^{\mathrm{b}, \mathrm{c}}$, Bhavya Rehani ${ }^{\mathrm{d}}$ \\ a Massachusetts General Hospital, Harvard Medical School, Boston, USA \\ ${ }^{\mathrm{b}}$ Department of Mechanical Engineering, Catholic University of Leuven, Leuven, Belgium \\ ${ }^{\mathrm{c}}$ Department of Radiology, Faculty of Dentistry, Chulalongkorn University, Bangkok, Thailand \\ d Department of Radiology and Biomedical Imaging, University of California, San Francisco, CA, USA
}

\section{A B S T R A C T}

Purpose: The main aim of medical physics training in radiology residency is to

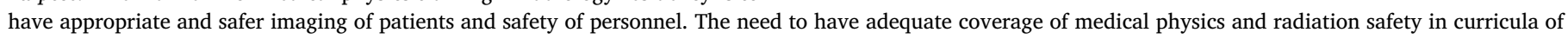
radiology residency is well perceived, but it is not known how far it is implemented in practice.


between countries in function of their human development index (HDI).

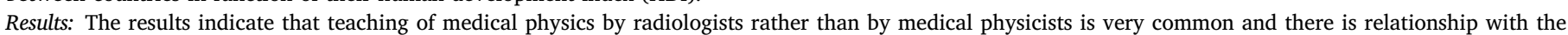

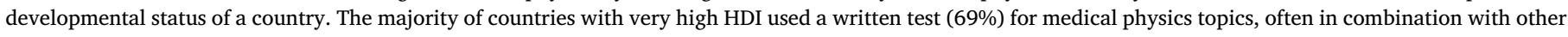

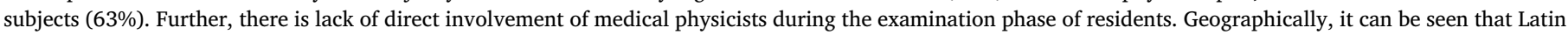
American countries in particular lack involvement of medical physicists during both the teaching and examination phase.

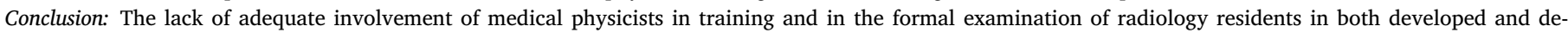
veloping countries is a matter of concern with likely implications on patient and staff safety.

\section{Introduction}

The important goal of medical physics training in radiology residency is to have appropriate and safer imaging of patients and safety of personnel. The breadth and depth of scientific knowledge underlying the practice of diagnostic radiology helps a practicing radiologist in understanding the strengths and limitations of the tools in their practice. The resident should become familiar with the technical aspects of image formation in each imaging modality, factors that impact image quality, balancing of image quality and radiation dose and thus achieving patient and staff safety. The pivotal role of medical physics training in radiology residency has been recognized for decades $[1,2]$ and the increasing complexity and enhanced utilization of imaging equipment involving radiation exposure have necessitated an enhanced role of medical physics training. Furthermore, it is necessary that the resident's knowledge, skills and competences in medical physics are properly assessed.

There are specific requirements of medical physics training to radiology residents in developed countries like US [1], UK [2,3] and in the European [4] region. The American Board of Radiology (ABR) and the Royal College of Radiologists (RCR) involve professional societies of medical physics [American Association of Physicists in Medicine
(AAPM) and Institute of Physics \& Engineering in Medicine (IPEM)] to determine the curriculum, to prepare the training material and seek participation of medical physicists (MPs) in the development of an evaluation system for board certification. A similar system prevails in Australia where the Royal Australian and New Zeeland College of Radiologists (RANZCR) and Australian College of Physical Scientists and Engineers in Medicine (ACPSEM) operate [5]. Unfortunately, a similar explicit role of MPs and corresponding national professional organizations is often lacking, also in the European Training Curriculum for Radiology.

The status of radiology education has recently been evaluated through a survey of 72 countries from four regions: Africa [6] Asia [7] Europe [8] and Latin America [9]. These papers did not analyze or cross-correlate specific information regarding the medical physics aspect such as inter-regional comparison as well as classification of countries by human development index [9], which is covered in this Technical Note.

\section{Material \& methods}

This study is a part of a worldwide survey on radiology education that spans countries in Africa, Asia, Latin America, and Europe [6-9].

\footnotetext{
* Corresponding author at: Radiology Department, Massachusetts General Hospital and Harvard Medical School, 175 Cambridge Str., Suite 244, Boston, MA 02114, USA.

E-mail address: madan.rehani@gmail.com (M.M. Rehani).
} 
The questions pertaining to medical physics included identifying if MPs or radiologists or both teach medical physics to radiology residents, if there is a separate question paper on this subject and if so, whether it is combined with another subject, and whether it is an oral and/or written test. Further questions inquired if radiologists or MPs review the answer sheets for medical physics portion and who conducts oral exam in medical physics and radiation safety. For intercomparing the countries, they were classified according to their Human Development Index (HDI), as defined by the 2016 Human Development Report of the United Nations Development Programme [10]. Also, categorization of the data was done per geographical region.

\section{Results}

The classification of countries included in this survey into HDI is:

- Very High HDI $(\mathrm{n}=27)$ : Argentina, Austria, Chile, Croatia, Denmark, Greece, Hong Kong SAR, Hungary, Israel, Italy, Japan, Lithuania, Malta, Norway, Poland, Portugal, Romania, Saudi Arabia, Serbia, Slovakia, Slovenia, South Korea, Spain, Sweden, Switzerland, United Arab Emirates, United Kingdom

- High HDI ( $\mathrm{n}=23)$ : Algeria, Armenia, Brazil, Bulgaria, China, Colombia, Costa Rica, Cuba, Dominican Republic, Ecuador, Iran, Kenya, Lebanon, Malaysia, Mexico, Oman, Panama, Peru, Sri Lanka, Thailand, Turkey, Uruguay, Venezuela

- Medium HDI $(\mathrm{n}=11)$ : Bangladesh, Egypt, El Salvador, Ghana, Guatemala, Honduras, India, Nicaragua, Pakistan, Philippines, South Africa

- Low HDI $(n=6)$ : Ethiopia, Nigeria, Senegal, Syria, Tanzania, Uganda

Table 1 shows that, in countries with a very high HDI, teaching of medical physics by radiologists was not so common ( $15 \%, n=4 / 27$ ), whereas in countries with low, medium or high HDI the teaching was solely performed by radiologists in $40 \%(n=2 / 5), 50 \%(n=5 / 10)$ and $41 \%(n=9 / 22)$ of the surveyed countries, respectively. In a small minority of the countries, teaching was performed by both radiologists and MPs. There was not so much difference in the extent of involvement of MPs in teaching among low, medium and high HDI countries with respective figures of $40 \%(\mathrm{n}=2 / 5), 50 \%(\mathrm{n}=5 / 10)$ and $55 \%(\mathrm{n}=12$ / 22 ), as compared to very high HDI countries where the figure was $74 \%$ ( $\mathrm{n}=20 / 27$ ).
In the group with very high HDI, most countries included a formal examination of medical physics during the radiology residency, usually involving a written test $(69 \%, \mathrm{n}=11 / 16)$ often in combination with other subjects $(63 \%, n=10 / 16)$. In the other HDI categories, an oral or combined oral/written test was more prevalent. In countries with medium, high or very high HDI, radiologists reviewed answers to medical physics questions in $27 \%(n=3 / 11), 38 \%(n=8 / 21)$ and $33 \%$ ( $n=9 / 27$ ) of the surveyed countries.

Some limitations of this analysis are apparent: the sample size of countries in HDI categories "low" $(n=6)$ and "medium" $(n=11)$ is small, which can skew percentage values. The sample size was further reduced due to blank answers provided to certain questions, as explained in Table 1.

When splitting up the surveyed countries by geographical location (Table 2), an interesting finding is the lower frequency of involvement of MPs during training in Latin American countries $(40 \%, \mathrm{n}=6 / 15)$ compared with Africa $(60 \%, n=6 / 10)$, Asia $(67 \%, n=12 / 18)$ and Europe $(71 \%, n=15 / 21)$. Furthermore, examination of medical physics was more often done through a written test in Latin America (82\%, $\mathrm{n}=9 / 11)$ and Europe $(58 \%, \mathrm{n}=7 / 12)$ than in Africa $(22 \%, \mathrm{n}=2 / 9)$ or Asia $(33 \%, n=5 / 15)$.

\section{Discussion}

To appreciate these findings, it is appropriate to start with the prevailing system in countries like UK, US and Australia. In these countries, there is a professional body of radiology (e.g. RCR, ABR, RANZCR). This body provides certification to radiologists after satisfactory completion of requirements, which also includes a medical physics curriculum. For the medical physics part, the body depends upon the medical physics experts provided by the corresponding society of the country for forming a committee to design the curriculum, prepare a question paper or question bank, and evaluate answers. Regular formal assessment is invariably recommended, with no specific requirements regarding the type of assessment or the background of the assessor(s). The UK curriculum specifically mentions a multiple choice question based assessment [2]. The multisource feedback (MSF) tool assesses generic skills across the domain of Good Medical Practice [3]. It consists of collated views from a range of co-workers (previously described as 360-degree assessment). For MSF, the UK curriculum recommends a group of assessors with a mixed background, without specifically mentioning MPs. US training includes a Physics category

Table 1

Overview of medical physics training in countries categorized by Human Development Index.

\begin{tabular}{|c|c|c|c|c|c|c|c|c|c|}
\hline & \multirow[t]{2}{*}{$\begin{array}{l}\text { Human Development Index } \\
\text { Number of countries }\end{array}$} & \multicolumn{2}{|l|}{$\begin{array}{l}\text { Low } \\
6\end{array}$} & \multicolumn{2}{|c|}{$\begin{array}{l}\text { Medium } \\
11\end{array}$} & \multicolumn{2}{|l|}{$\begin{array}{l}\text { High } \\
23\end{array}$} & \multicolumn{2}{|c|}{$\begin{array}{l}\text { Very high } \\
27\end{array}$} \\
\hline & & Freq. & Perc. & Freq. & Perc. & Freq. & Perc. & Freq. & Perc. \\
\hline \multirow[t]{4}{*}{ Medical physics taught by: } & Radiologist & 2 & $40 \%$ & 5 & $50 \%$ & 9 & $41 \%$ & 4 & $15 \%$ \\
\hline & MP & 2 & $40 \%$ & 5 & $50 \%$ & 12 & $55 \%$ & 20 & $74 \%$ \\
\hline & Both & 1 & $20 \%$ & 0 & $0 \%$ & 1 & $5 \%$ & 2 & $7 \%$ \\
\hline & Other & 0 & $0 \%$ & 0 & $0 \%$ & 0 & $0 \%$ & 1 & $4 \%$ \\
\hline \multirow[t]{8}{*}{ Formal examination: } & $\mathrm{No}^{\mathrm{a}}$ & 0 & $0 \%$ & 0 & $0 \%$ & 1 & $6 \%$ & 0 & $0 \%$ \\
\hline & Question paper with other subject ${ }^{\mathrm{a}}$ & 0 & $0 \%$ & 3 & $38 \%$ & 4 & $25 \%$ & 10 & $63 \%$ \\
\hline & Separate question paper ${ }^{\mathrm{a}}$ & 4 & $100 \%$ & 6 & $75 \%$ & 12 & $75 \%$ & 8 & $50 \%$ \\
\hline & Only oral test ${ }^{\mathrm{b}}$ & 1 & $17 \%$ & 1 & $10 \%$ & 1 & $7 \%$ & 3 & $19 \%$ \\
\hline & Only written test $\mathrm{t}^{\mathrm{b}}$ & 0 & $0 \%$ & 5 & $50 \%$ & 7 & $47 \%$ & 11 & $69 \%$ \\
\hline & Both oral and written test ${ }^{\mathrm{b}}$ & 5 & $83 \%$ & 4 & $40 \%$ & 7 & $47 \%$ & 2 & $13 \%$ \\
\hline & Radiologists review MP answers ${ }^{\mathrm{c}}$ & 0 & $0 \%$ & 3 & $27 \%$ & 8 & $38 \%$ & 9 & $33 \%$ \\
\hline & Radiologists conduct oral exam in $\mathrm{MP}^{\mathrm{c}}$ & 0 & $0 \%$ & 0 & $0 \%$ & 3 & $14 \%$ & 2 & $7 \%$ \\
\hline
\end{tabular}

\footnotetext{
${ }^{a}$ The percentage was calculated according to the total number of countries that provided at least one answer out of the three possible options that show this footnote. Countries were allowed to select both options (i.e. both a combined and separate paper); therefore, the total percentage may be higher than $100 \%$.

b The percentage was calculated according to the total number of countries that provided at least one answer out of the three possible options that show this footnote.

c The percentage was calculated according to the total number of countries that provided at least one answer for any option under the category 'formal examination'.
} 
Table 2

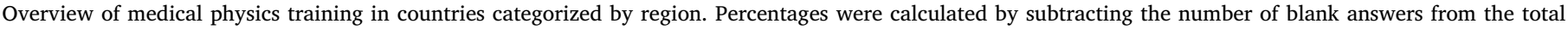
number of countries per region.

\begin{tabular}{|c|c|c|c|c|c|c|c|c|c|c|c|}
\hline & \multirow[t]{2}{*}{ Number of countries } & \multicolumn{2}{|l|}{$\begin{array}{l}\text { Africa } \\
10\end{array}$} & \multicolumn{2}{|l|}{$\begin{array}{l}\text { Asia } \\
19\end{array}$} & \multicolumn{2}{|c|}{$\begin{array}{l}\text { Europe } \\
21\end{array}$} & \multicolumn{2}{|c|}{$\begin{array}{l}\text { Latin America } \\
17\end{array}$} & \multicolumn{2}{|l|}{$\begin{array}{l}\text { Total } \\
67\end{array}$} \\
\hline & & Freq. & Perc. & Freq. & Perc. & Freq. & Perc. & Freq. & Perc. & Freq. & Perc. \\
\hline \multirow[t]{4}{*}{ Medical physics taught by: } & Radiologist & 3 & $30 \%$ & 5 & $28 \%$ & 5 & $24 \%$ & 7 & $47 \%$ & 20 & $31 \%$ \\
\hline & Medical Physicist & 6 & $60 \%$ & 12 & $67 \%$ & 15 & $71 \%$ & 6 & $40 \%$ & 39 & $61 \%$ \\
\hline & Both & 1 & $10 \%$ & 1 & $6 \%$ & 0 & $0 \%$ & 2 & $13 \%$ & 4 & $6 \%$ \\
\hline & Other & 0 & $0 \%$ & 0 & $0 \%$ & 1 & $5 \%$ & 0 & $0 \%$ & 1 & $2 \%$ \\
\hline \multirow[t]{8}{*}{ Formal examination: } & $\mathrm{No}^{\mathrm{a}}$ & 1 & $10 \%$ & 0 & $0 \%$ & 0 & $0 \%$ & 0 & $0 \%$ & 1 & $2 \%$ \\
\hline & Question paper with other subject ${ }^{\mathrm{a}}$ & 0 & $0 \%$ & 8 & $50 \%$ & 8 & $67 \%$ & 1 & $13 \%$ & 17 & $39 \%$ \\
\hline & Separate question paper ${ }^{\mathrm{a}}$ & 7 & $88 \%$ & 11 & $69 \%$ & 5 & $42 \%$ & 7 & $88 \%$ & 30 & $68 \%$ \\
\hline & Only oral test ${ }^{\mathrm{b}}$ & 0 & $0 \%$ & 3 & $20 \%$ & 3 & $25 \%$ & 0 & $0 \%$ & 6 & $13 \%$ \\
\hline & Only written test $\mathrm{t}^{\mathrm{b}}$ & 2 & $22 \%$ & 5 & $33 \%$ & 7 & $58 \%$ & 9 & $82 \%$ & 23 & $49 \%$ \\
\hline & Both oral and written test ${ }^{\mathrm{b}}$ & 7 & $78 \%$ & 7 & $47 \%$ & 2 & $17 \%$ & 2 & $18 \%$ & 18 & $38 \%$ \\
\hline & Radiologists review MP answers $^{c}$ & 0 & $0 \%$ & 5 & $26 \%$ & 7 & $33 \%$ & 8 & $47 \%$ & 20 & $30 \%$ \\
\hline & Radiologists conduct oral exam in $\mathrm{MP}^{\mathrm{c}}$ & 0 & $0 \%$ & 1 & $5 \%$ & 1 & $5 \%$ & 3 & $18 \%$ & 5 & $7 \%$ \\
\hline
\end{tabular}

a The percentage was calculated according to the total number of countries that provided at least one answer out of the three possible options that show this footnote. Countries were allowed to select both options (i.e. both a combined and separate paper); therefore, the total percentage may be higher than 100\%.

b The percentage was calculated according to the total number of countries that provided at least one answer out of the three possible options that show this footnote.

c The percentage was calculated according to the total number of countries that provided at least one answer for any option under the category 'formal examination'.

during Core Examination (currently taken 36 months after starting the radiology residency) [1]. Previously, the physics exam used to be after first year. The ABR core examination for radiologists contains material on medical physics. This content is based on the medical physics that is used in practice by working radiologists in academic and private practice [1]. Computer-based examination is used in the US, which can help in assessing multiple choice questions (MCQs) automatically without physical need for specific expertise. The involvement of MPs in oral tests is also envisaged. The RANZCR and European (ESR) curricula have much less focus on assessment for medical physics part as such and there is tendency to merge with imaging modality which may be sensible from the subject perspective but may not be optimal if medical physics part is covered by the radiologist.

While many countries at different levels of HDI have radiology education/residency programs, they are not matched with a similar level of support of medical physics as in UK or US. The lack of MPs in diagnostic radiology and thus their involvement or minimal participation in radiology education and assessment in such countries can raise questions on the potential impact on patient and staff safety $[11,12]$. As a matter of nomenclature, medical physics in this paper included radiation protection (RP) of patients and staff.

Training material targeted at residents has been made available by the Radiological Society of North America (RSNA) and AAPM [13] and is available to members of either organization. A similar situation exists for training materials by other organizations. The IAEA has a tradition of making training materials in different areas publicly available, but they do not have material targeted at radiology residents specifically and the material in any particular area is not coupled with an examination system. The European Training Curriculum for Radiology [4] has modules on Radiation Protection Education and Training. The medical physics topics are included for each imaging modality as mentioned above. The modules are foreseen in Level I training (years 1-3). However, the training material has partly been developed for radiation protection and not for medical physics as a whole. The access to training material is limited to members.

The magnitude of lack of involvement of MPs in both teaching and evaluation is apparent in a large part of the world. While actions at developing a standard curriculum for developing countries may be feasible, major issues with assessment will remain for countries that do not have a local system; such countries may need to accept an assessment by an external organization which may be a much more viable option technically and financially till the country reaches a stage of selfreliance.

The International Commission on Radiological Protection (ICRP) in its Publication 113 [14] states that a training programme in RP for healthcare professionals has to be oriented towards the type of training to which the target audience is accustomed, and the presentations should be tailored to clinical situations to impart skills in the appropriate context. Further, the primary trainer in RP should be a person who is an expert in RP in the practice with which they are dealing. This means a person who, in addition to having a detailed understanding of radiological protection, has knowledge about the clinical practice in the use of radiation. Different aspects of the role of MPs in education of healthcare professionals in Europe have been deliberated [15-17]. The shortage of diagnostic MPs in large parts of the world has been pointed out by the IAEA $[11,12]$. Another aspect that needs consideration is the integration of MPs in day-to-day radiological practice. In previous years, MPs were equipment-focused, dealing with quality control (QC) testing. The transition from equipment to patient dose brought MPs closer to clinical practice. In recent years developments in dose management systems are providing easy remote access to dose information to MPs. While that increases access and provides more information, it reduces physical interactions with radiology colleagues in the department. The bottom-line seems to be the usefulness of MPs to colleagues in the department. Much of this comes through radiation protection. A SWOT analysis of the role of the MPs in the education of healthcare professionals in Europe presents strengths and weaknesses [16] and there have been aspects of strategic developments on enhancing the role of MPs [17]. Further the European directive requires radiation protection and medical physics training to healthcare professionals [18]. Although it is difficult to find published information, there seems to be a tendency that residents tend to prefer preparing for exam just before the examinations rather than attending structured classes in subjects like medical physics throughout their residency period. There is need to assess this practice vis-à-vis other subjects. This tendency to use medical physics only to pass the exam is probably not restricted to medical physics but a cultural issue. The regulations can require assessment of knowledge, but professional guidelines need to require skill and competence assessment [18]. The drive to automated assessment using multiple choice questions proves sub-optimal for skill and competence assessment.

What can be the way forward? We are in the era of artificial 
intelligence, remote education and remote assessment. This leads us to globalization of education. The globalization in various aspects of daily life has led to 'survival of fittest' products wherein trademarks with good standing started to rule. High-quality technology-based solutions, not only restricted to e-learning but comprising assessment through the internet as well, are needed to remedy the situation. It is not possible to depend upon local resources in large parts of the world to fill the gaps in human resources, both in number and competencies. There is certainly no denying of the fact that face to face interactions are important and should be encouraged.

In summary, there is a clear need for MPs to be more involved in medical physics teaching during radiology residency and in formal written and oral examination of radiology residents. Technology-based solutions may be one of the several options to remedy the situation.

\section{References}

[1] American Board of Radiology. Preparing for Medical Physics Components of the ABR Core Examination. https://www.theabr.org/sites/all/themes/abr-media/pdf/ Physics.pdf.

[2] The Royal College of Radiologists. Structured Training in Clinical Radiology. Fourth Edition. London. https://www.rcr.ac.uk/sites/default/files/docs/radiology/pdf/ STCR-2004.pdf.

[3] Royal College of Radiologists. Specialty Training Curriculum for Clinical Radiology. Edition December 2016, https://www.rcr.ac.uk/sites/default/files/cr_curriculum2016_final_15_november_2016_0.pdf.

[4] European Society for Radiology. Revised European Training Curriculum for Radiology. Edition March 2016, http://www.myesr.org/sites/default/files/ESR 2016_ESR-EuropeanTrainingCurriculum_LEVEL_I\%2BII_Edition_March_2016.pdf.

[5] The Royal Australian and New Zealand College of Radiologists. Clinical Radiology (Radiodiagnosis) Training Program Curriculum. Edition October 2017. https:// www.ranzcr.com/trainees/clinical-radiology/curriculum.

[6] Rehani B, Brown I, Dandekar S, Sarkodie B, Mwango G, Rehani MM, et al. Radiology
Education in Africa: Analysis of Results From 13 African Countries. J Am Coll Radiol. 2017;14:247-52.

[7] Rehani B, Gao KT, Lau L, Rehani MM, Zhang YC, Dillon WP. Radiology Education in Asia: Differences, Similarities, and Opportunities. J Am Coll Radiol. 2017;14:111-8.

[8] Rehani B, Zhang YC, Rehani MM, Palkó A, Lau L, Lette MN, et al. Radiology education in Europe: analysis of results from 22 European countries. World J Radiol. 2017;9:55-62.

[9] Rehani B, Zhang YC, Gao KT, Soto Giordani G, Lau L, Rehani MM, et al. Radiology Education in Latin America. J Am Coll Radiol. 2017;14:397-403.

[10] United Nations Development Programme. Human Development Report 2016: Human Development for Everyone, http://hdr.undp.org/en/2016-report/ download.

[11] Clinical Training of Medical Physicists Specializing in Diagnostic Radiology. Training Course Series No. 47, Vienna: IAEA; 2010

[12] Medical Physics Staffing Needs in Diagnostic Imaging and Radionuclide Therapy: An Activity Based Approach. IAEA Human Health Reports No. 15, Vienna: IAEA; 2018.

[13] RSNA/AAPM Online Physics Modules. http://www.rsna.org/physics-modules.

[14] Vañó E, Rosenstein M, Liniecki J, Rehani MM, Martin CJ, Vetter RJICRP. Publication 113. Education and training in radiological protection for diagnostic and interventional procedures. Ann ICRP. 2009 Oct;39(5):7-68. https://doi.org/10. 1016/j.icrp.2011.01.002.

[15] Caruana CJ, Wasilewska-Radwanska M, Aurengo A, Dendy PP, Karenauskaite V, Malisan MR, et al. The role of the biomedical physicist in the education of the healthcare professions: an EFOMP project. Phys Med. 2009 Sep;25(3):133-40. https://doi.org/10.1016/j.ejmp.2008.11.001.

[16] Caruana CJ, Wasilewska-Radwanska M, Aurengo A, Dendy PP, Karenauskaite V, Malisan MR, et al. A comprehensive SWOT audit of the role of the biomedical physicist in the education of healthcare professionals in Europe. Phys Med. 2010 Apr;26(2):98-110. https://doi.org/10.1016/j.ejmp.2009.08.001.

[17] Caruana CJ, Wasilewska-Radwanska M, Aurengo A, Dendy PP, Karenauskaite V, Malisan MR, et al. A strategic development model for the role of the biomedical physicist in the education of healthcare professionals in Europe. Phys Med. 2012 Oct;28(4):307-18. https://doi.org/10.1016/j.ejmp.2011.05.001.

[18] Caruana CJ, Tsapaki V, Damilakis J, Brambilla M, Martín GM, Dimov A, et al. and others. EFOMP policy statement 16: the role and competences of medical physicists and medical physics experts under 2013/59/EURATOM. Phys. Med. 2018;48:162-8. 